\section{IN THE NEWS}

Cancer vaccine success An experimental vaccine is proving effective at protecting against cervical cancer and genital warts, according to a new study published in The Lancet Oncology (Reuters).

The vaccine - Gardasil - is being developed by Merck \& Co., and it targets four strains of human papillomavirus (HPV): HPV16 and HPV18, which are associated with $\sim 70 \%$ of cervical cancers; and HPV6 and HPV11, which cause genital warts. Worldwide, cervical cancer affects approximately half a million women each year, and of these, about half will die (Associated Press). The Merck trial involved $\sim 500$ women from Brazil, the United States and Europe, half of whom received the vaccine. The trial participants were then tested for HPV infection and lesions or genital warts during a 2.5-year period. According to Dr Luisa Villa of the Ludwig Institute for Cancer Research in Brazil, "If [larger] Phase III studies demonstrate the vaccine is as effective as this, I'm sure that it will change the history of cervical cancer" (Reuters).

A rival vaccine -

Cervarix - that targets the two strains of HPV that are associated with cervical cancer is being developed by GlaxoSmithKline and is at a similar stage of development. "The race is on. There is not much to choose between these vaccines. In practice both have proved to be extremely effective", said Dr Anne Szarewski, a clinical consultant at Cancer Research UK (The Daily Telegraph, UK). Both vaccines are expected to reach the market within the next two to three years.

Elaine Bell

\title{
IMMUNE REGULATION
}

\section{Co-stimulation with TIMs}

Two papers published in the May issue of Nature Immunology provide insight into the function and specificity of the newly identified TIM ( $\mathrm{T}$-cell immunoglobulin domain and mucin domain) family of proteins and highlight their role in regulating $\mathrm{T}$-cell responses.

The TIM gene family has eight members in mice and three in humans, all of which are predicted to encode transmembrane proteins that have an IgV domain, a mucin-like domain and a cytoplasmic tail. Umetsu et al. focused their studies on the function of TIM1, whereas Meyers et al. sought to elucidate the role of TIM4 in the immune system.

Using a monoclonal antibody specific for mouse TIM1, Umetsu et al. first showed that TIM1 was expressed by activated $\mathrm{T}$ cells and by $\mathrm{T}$ cells cultured in T helper $2\left(\mathrm{~T}_{\mathrm{H}} 2\right)$-cell-polarizing conditions, and it was absent or present at low levels in resting $\mathrm{T}$ cells and $\mathrm{T}_{\mathrm{H}} 1$ cells, respectively. By contrast, Meyers et al. showed that TIM4 was not expressed by T cells but was expressed by antigen-presenting cells, with the highest expression levels by mature dendritic cells of the lymphoid lineage. To identify the ligand for TIM4, Meyers et al. generated a TIM4-immunoglobulin fusion protein. The fusion protein specifically bound activated (but not resting) $\mathrm{B}$ and T cells, which led the authors to propose that the TIM4 ligand could be TIM1. To confirm this, they showed that the TIM4 fusion protein bound TIM1expressing cells, and conversely, a TIM1 fusion protein bound TIM4-expressing cells but not untransfected cells or cells expressing other TIM-family members. Moreover, consistent with the TIM1 expression pattern described by Umetsu et al., the TIM4 fusion protein showed a higher level of binding to activated $\mathrm{T}_{\mathrm{H}} 2$ cells than to $\mathrm{T}_{\mathrm{H}} 1$ cells.
Next, both groups sought to determine the effect of TIM1 ligation using either the TIM1-specific antibody or the TIM4 fusion protein. In vitro, TIM1-specific antibody increased the proliferation of $\mathrm{CD}^{+} \mathrm{T}$ cells stimulated with antibodies specific for CD3 and CD28. It also increased cytokine production by $\mathrm{T}_{\mathrm{H}} 2$ cells but had no effect on $\mathrm{T}_{\mathrm{H}} 1$ cells, which do not express TIM1. T cells from T-cell-receptor-transgenic mice treated with TIM1specific antibody and primed with the cognate antigen had increased levels of both basal proliferation and proliferation in response to antigen exposure in vitro. These cells also produced more interferon- $\gamma$, interleukin-4 (IL-4) and IL-10. Similarly, Meyers et al. showed that in vivo administration of the TIM4 fusion protein increased proliferation of primed T cells, with or without antigen re-stimulation. In addition, in vivo ligation of TIM4 by administration of the TIM1 fusion protein increased in vitro $\mathrm{T}$-cell proliferation and promoted the production of $\mathrm{T}_{\mathrm{H}} 2$ cytokines in favour of $\mathrm{T}_{\mathrm{H}} 1$ cytokines, which would usually be produced in the mouse model used.

Finally, Umetsu et al. showed that treatment with TIM1-specific antibody could break tolerance to inhaled allergen and promote the development of airway hyper-reactivity after allergen challenge, which is consistent with the identification of Tim1 as an asthma-susceptibility gene.

Lucy Bird

\section{(Q) References and links}

ORIGINAL RESEARCH PAPERS Umetsu, S. E. et al. TIM-1 induces T cell activation and inhibits the development of peripheral tolerance. Nature Immunol. 6, 447-454 (2005) | Meyers, J. H. et al. TIM-4 is the ligand for TIM-1, and the TIM-1-TIM-4 interaction regulates T cell proliferation. Nature Immunol. 6, 455-464 (2005)

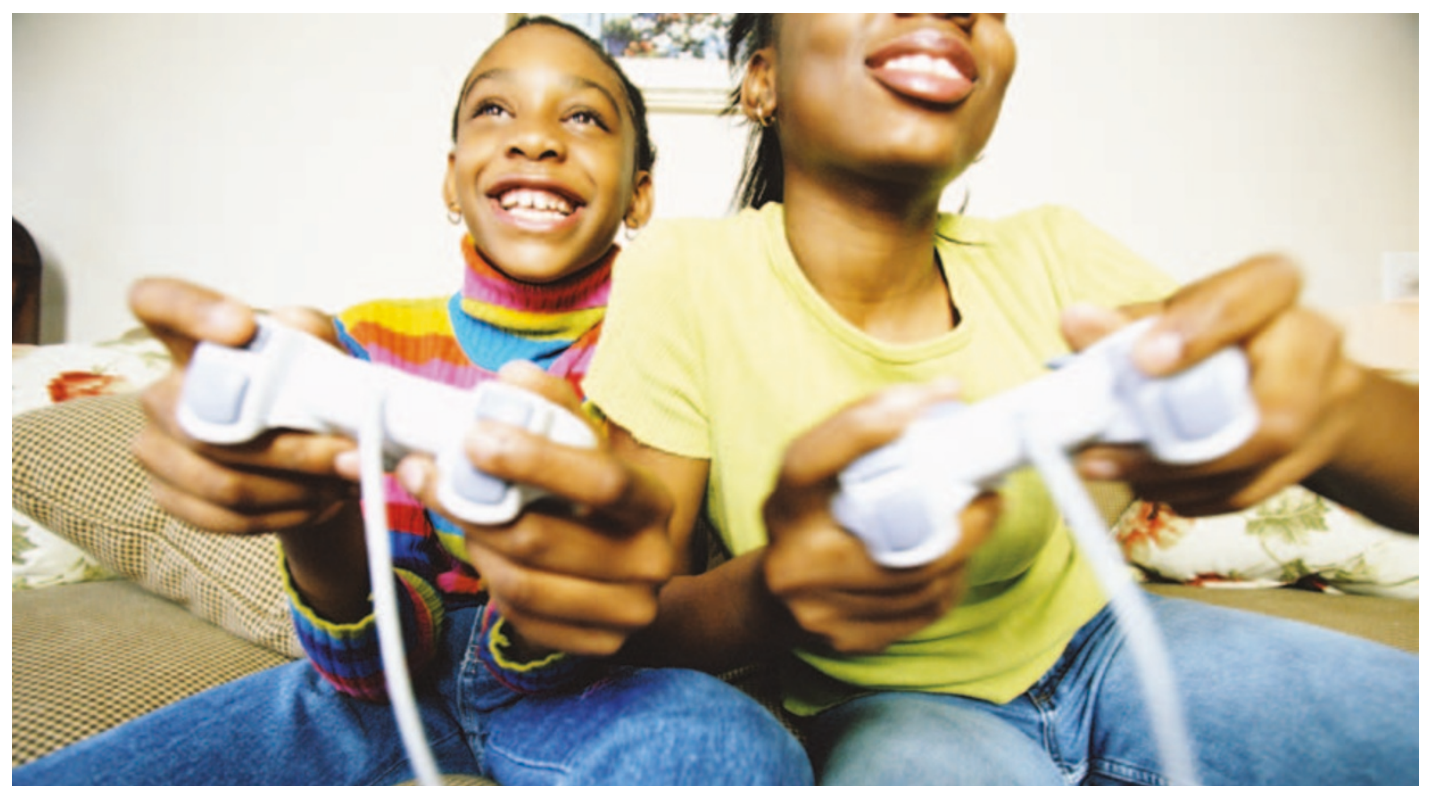

